Blucher

Blucher Proceedings

Cuba e Brasil no Século XXI (CBS21)

A Construção do Conhecimento

\title{
El Estímulo a la Ciencia, la Innovación y los Servicios Científico-Técnicos
}

\author{
Dr. Augusto González Garcia ${ }^{1}$
}

\section{Presentación}

El presente artículo se basa en un breve reporte mío para la Sociedad Cubana de Física $^{2}$, de la visita de una delegación de profesores, científicos y funcionarios cubanos, de la cual formé parte, que estuvo en las ciudades brasileras de Recife y Maceió del 28 de Julio al 6 de Agosto de 2013. La actividad constituyó la segunda etapa de la iniciativa CBS21.

\section{Encuentro Cuba-Brasil en el Siglo XXI - CBS21}

Dándole continuidad al encuentro Cuba-Brasil en el siglo XXI (CBS21), desarrollado en La Habana del 24 al 28 de Sept. de 2012 y promovido por los profesores Hugo Pérez Rojas (ICIMAF, Cuba) y César Zen Vasconcelos (UFRGS, Brasil) ${ }^{3}$, una delegación de 23 profesores, científicos y funcionarios visitó del 28 de Julio al 6 de Agosto las ciudades de Recife, Olinda y Maceió.

Durante la visita se produjeron múltiples encuentros con partners brasileros y autoridades locales y asistimos a centros de interés. Los recursos para esta actividad fueron gestionados por César Zen Vasconcellos y Josealdo Tonholo (UFAL,

\footnotetext{
${ }^{1}$ Ex-Presidente de la Sociedad Cubana de Física (SCF), Instituto de Cibernética, Matemática y Física (ICIMAF), La Habana, Cuba.

E-mail: agonzalecu@gmail.com.

${ }^{2}$ www.fisica.uh.cu/scf/index.php/en/reportes/83 - reportes/207 - cbs $21-2$

${ }^{3}$ www.fisica.uh.cu/scf/index.php/en/noticias/89 - noticias $2 / 142-$ sesiona - cbs 21
} 
Brasil) ante el CNPq, otras fundaciones brasileras de apoyo a la ciencia, la educación y la industria así como los gobiernos de los estados de Pernambuco (capital - Recife) y Alagoas (capital - Maceió).

Los estados de Pernambuco y Alagoas radican en el nordeste de Brasil, la región mas atrasada del pais. Al decir del Secretario de Ciencia y Tecnologia de Alagoas, la mitad de la población de esa región se haya por debajo del límite de pobreza y la cuarta parte por debajo del límite de pobreza extrema. Asimismo, la tasa de analfabetismo es altísima.

Paradójicamente, las economías de estos estados experimentan los índices mas altos de crecimiento de Brasil. Alli existen programas de apoyo a la ciencia, la educación, las empresas. El lenguaje de las autoridades es coherente. Requieren que el crecimiento económico se traduzca en desarrollo social, se preocupan por la inclusión de los sectores marginados de la población y porque el progreso se extienda por todo el país, dejando de concentrarse en las grandes ciudades.

Entre los centros visitados, me causó gran impresión el puerto de Suape ${ }^{4}$, alrededor del cual se instalan a ritmo vertiginoso empresas constructoras de automóviles, productoras de cemento y otros materiales de construcción, navieras, refinadoras de petróleo, empresas metalúrgicas, de alimentos, etc. Tengo la esperanza de que las autoridades nuestras a cargo de la construcción del puerto del Mariel estudien minuciosamente estas experiencias. Los directivos del puerto cubano fueron invitados a integrar la delegación aunque, por razones que desconozco, no asistieron.

Otra de las instituciones presentadas a la delegación fue el denominado Puerto Digital en Recife $\mathrm{e}^{5}$ - novedoso en extremo, fuera de lo comun. Esta es una iniciativa que, sobre la base de las modernas tecnologías de la informática, aglutina instalaciones educativas, culturales, pequeñas empresas, etc. Se localiza en la parte antigua de la ciudad, en locales restaurados. Segun su director, en algún momento el Puerto Digital tomará el liderazgo en Recife, cuando el puerto de Suape, el mas dinámico actualmente, frene su crecimiento por falta de espacio y recursos naturales.

\section{Algunas Ideas Sobre la Situación de la Ciencia y la Innovación en Cuba}

Lo vivido por esos dias en los estados de Pernambuco y Alagoas, me estimula a compartir algunas ideas sobre la situación de la Ciencia y la innovación en Cuba. En primer lugar, no arrastramos el lastre que significa el $50 \%$ de la población con problemas serios de acceso a la educación, la salud y la cultura en general. Nuestro

\footnotetext{
${ }^{4}$ http : //www.suape.pe.gov.br/news/matLer.php?id $=205$

${ }^{5}$ http : //www.portodigital.org/
} 
punto de partida, gracias a la Revolución, es mucho mas alto. Tenemos una red de centros de educación superior y de ciencia distribuídos por todo el país.

Contamos con un millón de graduados universitarios, aproximadamente el 10\% de la población. Propiciar la interacción entre ellos a partir de una red nacional de internet no significaría poner a ebullir la creatividad y la innovación en Cuba? Por el contrario, la medida reciente de hacer extensiva la Internet a $4.50 \mathrm{CUC}$ la hora establece una barrera al desarrollo de este millón de profesionales. El tráfico nacional de internet, que se puede separar del internacional, bien podría ofrecerse a otros costos, 4.50 CUP la hora por ej., para avivar, incentivar nuestras infinitas reservas de innovación.

Las trabas que impiden el desarrollo de las empresas estatales cubanas están cayendo una a una. Recientemente, a las empresas se les ha dado la posibilidad de manejar autónomamente el $50 \%$ de sus ganancias para inversiones y retribución a sus trabajadores. El polo cientifico de Biotecnología (BioFarmaCuba) optó por convertirse en una empresa, por lo que cuenta con estas prerrogativas. Pero, cuáles son los estímulos y apoyos al resto de nuestra ciencia, que por lo demás está descapitalizada? ${ }^{6}$

Hay signos de que se ha identificado el área de las energías renovables como de gran interés y perspectivas para nuestro país y que se comienzan a inyectar recursos importantes para el desarrollo de la industria relacionada con la misma. La labor de promoción y convencimiento desarrollada por Daniel Stolik, ex-presidente de la Sociedad Cubana de Física, es probable que haya jugado un papel en este escenario.

Sería importante que también se destinaran recursos a las instituciones de ciencia cuyo actuar se relaciona con las energías renovables y, en particular, las que llevan a cabo investigaciones de caracter básico en esta área. Aquí existen potencialidades de crear un segundo polo científico basado en desarrollos propios si se estimularan propiamente los grupos de investigación y se le asignaran recursos. El esquema del polo biotecnológico, donde primero fueron centros de investigación y después empresas, podría incluso invertirse y que sean los desarrrollos en las industrias de energías renovables los que jalen y exijan a la ciencia resultados propios que puedan eregirse en la base de la industria.

Por otro lado, las potencialidades de los servicios científico-técnicos son muy grandes. Los servicios técnicos y de innovación a empresas e instituciones extranjeras podrían convertirse en una fuente importante de ingresos para nuestra economía. Entonces, qué se necesita?: estímulos a las personas y a las instituciones. Si a las empresas ya se le ofreció el manejo autónomo del $50 \%$ de las ganancias, a los centros de ciencia y educación superior tal vez se le debería ofrecer mas. Conocemos por ej., que en la Escuela Politécnica de Zurich funciona una

\footnotetext{
${ }^{6}$ Informe sobre el estado de la Ciencia en Cuba, Reporte interno de la Academia de Ciencias de Cuba, Carlos Rodríguez et. al., Enero de 2013.
} 
fórmula simple que, extrapolada a nuestra realidad, sería asi: 1/3 de las ganancias para estimular a los responsables directos, $1 / 3$ para inversiones de la institución y $1 / 3$ para el presupuesto estatal.

Otro sector muy dinámico en Brasil es el de las pequeñas empresas de iniciativa privada basadas en la ciencia y la tecnología. Nosotros hemos comenzado a desarrollar el cuentapropismo y las cooperativas, tenemos un sector numéricamente grande de la población calificada, sabemos que la ciencia y la tecnología añaden valor agregado a las producciones, pero aun no hemos incentivado estas actividades. Un ejemplo clásico es el de la Universidad de Ciencias Informáticas (UCI), donde se han formado miles de programadores de nivel universitario, los cuales se encuentran realizando labores muy por debajo de su capacidad.

Por otro lado, desde hace dos años, como iniciativa de la Sociedad Cubana de Física, estamos adelantando una propuesta de consultoría a empresas, Innova.cu, donde podrían participar físicos, químicos, matemáticos, cibernéticos, etc. La respuesta siempre ha sido que no se están autorizando estas actividades. Acaso no es contradictorio?

Nuestro gobierno está empeñado en poner orden y disciplina en la economía. Esto es vital, sobre todo a corto plazo. La incorporación de la ciencia en la economía y la vida social tienen implicaciones a corto plazo porque dinamiza los ritmos de crecimiento y a largo plazo porque garantiza el futuro. Por eso es imprescindible y apremiante el estímulo a la Ciencia en Cuba. 